\title{
Equitable colorings of bounded treewidth graphs
}

Hans L. Bodlaender

Fedor V. Fomin

institute of information and computing sciences, utrecht university

technical report UU-CS-2004-010

www.cs.uu.nl 


\title{
Equitable colorings of bounded treewidth graphs
}

\author{
Hans L. Bodlaender* Fedor V. Fomin ${ }^{\dagger}$
}

\begin{abstract}
A proper coloring of a graph $G$ is equitable if the sizes of any two color classes are differ by at most one. The related notion is $\ell$-bounded coloring where each of the color classes is of cardinality $\leq \ell$. We consider the problems to determine for a given graph $G$ (and a given integer $\ell$ ) whether $G$ has an equitable ( $\ell$-bounded) coloring. We prove that both problems can be solved in polynomial time on graphs of bounded treewidth.
\end{abstract}

\section{Introduction}

There is a wide believe that almost every natural hard problem can be solved efficiently on graphs of bounded treewidth. Of course this is not true, a nice example is the BANDWIDTH MINIMIZATION problem which is NP hard even on trees of degree three [11, 25]. Another part of 'folklore' in Graph Algorithms community is that if some (natural) problem can be solved in polynomial time on trees, one should be able to solve it in polynomial time on graphs of bounded treewidth. However, there are some striking and frustrating examples, like $L(2,1)$-COLORING, when efficient algorithm for trees can be constructed [7] and nothing is known about the complexity of the problem on graphs of treewidth $\geq 2$. For more than ten years, EQUITABLE COLORING and $\ell$-BOUNDED COLORING were also examples of such problems. Both problems can be solved in polynomial time on trees and forests $[9,2,17]$, i.e. graphs of treewidth 1 and existence of a polynomial time algorithm for graphs of treewidth $\geq 2$ was an open question. In this paper we introduce the first polynomial time algorithm on graphs of bounded treewidth for both versions of coloring. Due to enormous exponents in the running our algorithm is mainly of theoretical interest. Our main technique is quite far from the standard dynamic programming on graphs of bounded treewidth. To convince the reader (and ourselves) that the standard dynamic programming approach is unlikely to implemented for EQUITABLE COLORING on graphs of bounded treewidth, we prove that a pre-colored version of the problem is NP hard on graphs of treewidth 1, i.e. forests. The

*Institute of Information and Computing Sciences, Utrecht University, 3508 TA Utrecht, The Netherlands, hansb@cs.uu.nl

${ }^{\dagger}$ Department of Informatics, University of Bergen, N-5020 Bergen, Norway, fomin@ii.uib.no 
main idea behind our polynomial time algorithm is to use recent combinatorial results of Kostochka et al. [21] that allow us to handle graphs with 'large' vertex degrees separately.

Previous results. The EqUITABLE COLORING problem has a long history. The celebrated theorem of Hajnal \& Szemerédi [13] says that any graph $G$ has an equitable $k$-coloring for $k \geq \Delta(G)+1$. This bound is sharp. One of the direction of research in this field was in obtaining better upper bounds than $\Delta(G)+1$ for special graph classes. See the survey [23] for a review of the results in this field.

The COLORING problem can be trivially reduced to EQUITABLE COLORING problem and thus EQUitable COLORING is NP hard. Polynomial time algorithms are known for split graphs [8] and trees [9].

$\ell$-BOUNDED COLORING has a number of applications. It is also known as the MUTUAL EXCLUSION SCHEDULING problem (MES) which is the problem of scheduling unit-time tasks non-preemptively on $m$ processors subject to constraints, represented by a graph $G$, so that tasks represented by adjacent vertices in $G$ must run in disjoint time intervals. This problem arises in load balancing the parallel solution of partial differential equations by domain decomposition. (See $[2,26]$ for more information.) Also the problems of this form have been studied in the Operations Research literature [3, 22]. Other applications are in scheduling in communication systems [15] and in constructing school timetables [19].

The $\ell$-BOUNDED $k$-COLORING problem can be solved in polynomial time on split graphs, complements of interval graphs [24,8], forests and in linear time on trees [2, 17]. This is almost all what is known on graph classes where the $\ell$-BOUNDED COLORING problem is efficiently solvable. When one of the parameters $\ell$ or $k$ is fixed the situation is different. For example, for fixed $\ell$ or $k$ the problem is solved on cographs [4, 24] and for fixed $\ell$ on bipartite graphs $[4,14]$ and line graphs [1]. For $\ell=2$ the problem is equivalent to the MAXIMUM MATCHING problem on the complement graphs and is polynomial. Notice that for fixed $\ell$ the problem can be expressed in the counting monadic second-order logic and for graphs of bounded treewidth linear time algorithm for fixed $\ell$ can be constructed [18]. When $\ell$ is not fixed (i.e. $\ell$ is part of the input) even for trees the situation is not simple and the question on existence of a polynomial time algorithm on trees [14] was open for several years.

The problem remains NP-complete on cographs, bipartite and interval graphs [4], on cocomparability graphs and fixed $\ell \geq 3$ [24], on complements of line graphs and fixed $\ell \geq 3$ [10], and on permutation graphs and $\ell \geq 6$ [16]. For $k=3$ the problem is NP-complete on bipartite graphs [4].

Almost all NP-completeness results for $\ell$-BOUNDED $k$-COLORING for different graph classes mentioned above can also be obtained for EQUITABLE $k$-COLORING by making use of the following observation.

Proposition 1.1. A graph $G$ on $n$ vertices is $\ell$-bounded $k$-colored if and only if the graph $G^{\prime}$ obtained from $G$ by adding an independent set of size $\ell k-n$ is equitable $k$-colorable.

Our contribution. A standard dynamic programming approach for the COLORING problem needs to keep $O\left(w^{k} n\right)$ entries, where $w$ is the treewidth of a graph and $k$ is the number 
of colors. Since the chromatic number of a graph is at most $w+1$ this implies that the classical coloring problem can be solved in polynomial time on graphs of bounded treewidth. Clearly such a technique does not work for EQUITABLE COLORING because the number of colors in an equitable coloring is not bounded by a function of treewidth. For example, a star on $n$ vertices has treewidth 1 and it can not be equitable $k$-colored for any $k<(n-1) / 2$. One of the indications that the complexity of EQUITABLE COLORING for graphs of bounded treewidth can be different from 'classical' is that by Proposition 1.1 and [4], the problem is NP hard on cographs and thus on graphs of bounded clique-width. (Note that chromatic number is polynomial on graphs of bounded clique-width [20].)

However, one of the properties of equitable colorings making our approach possible is the phenomena observed first by Bollobás \& Guy [5] for trees: 'Most' trees can be equitable 3 -colored. In other words, for almost all trees the difference between the numbers of colors in equitable coloring is not 'far' from the chromatic number. Recently Kostochka et al. [21] succeed to generalize Bollobás \& Guy result for degenerated graphs and our main contribution - the proof that EQUITABLE COLORING can be solved in polynomial time on graphs of bounded treewidth (Section 3) - strongly uses this result. Very roughly, we use the results of Kostochka et al. to establish the threshold when the problem is trivially solved and when it become to be solvable in polynomial time by dynamic programming developed in Section 2. In Section 4 we show that such an approach can not be extended to PRE-COLORED EQUITABLE COLORING by showing that the pre-colored version of the problem is NP hard on graphs of treewidth 1, i.e., forests.

\subsection{Definitions}

We denote by $G=(V, E)$ a finite undirected and simple graph. We usually use $n$ to denote the number of vertices in $G$. For every nonempty $W \subseteq V$, the subgraph of $G$ induced by $W$ is denoted by $G[W]$. The maximum degree of $G$ is $\Delta(G):=\max _{v \in V} d_{G}(v)$. A graph $G$ is $d$-degenerate if each of its nonempty subgraphs has a vertex of degree at most $d$. A nonempty subset of vertices $I \subseteq V$ is independent in $G$ if no two of its elements are adjacent in $G$.

Definition 1.2. A tree decomposition of a graph $G=(V, E)$ is a pair $\left(\left\{X_{i} \mid i \in I\right\}, T=\right.$ $(I, F))$, with $\left\{X_{i} \mid i \in I\right\}$ a family of subsets of $V$ and $T$ a tree, such that

- $\bigcup_{i \in I} X_{i}=V$.

- For all $\{v, w\} \in E$, there is an $i \in I$ with $v, w \in X_{i}$.

- For all $i_{0}, i_{1}, i_{2} \in I$ : if $i_{1}$ is on the path from $i_{0}$ to $i_{2}$ in $T$, then $X_{i_{0}} \cap X_{i_{2}} \subseteq X_{i_{1}}$.

The width of tree decomposition $\left(\left\{X_{i} \mid i \in I\right\}, T=(I, F)\right)$ is $\max _{i \in I}\left|X_{i}\right|-1$. The treewidth of a graph $G$ is the minimum width of a tree decomposition of $G$.

Lemma 1.3 (Folklore). Every graph on $n$ vertices and of treewidth $\leq w$ has at most wn edges. 
A $k$-coloring of the vertices of a graph $G=(V, E)$ is a partition $A_{1}, A_{2}, \ldots, A_{k}$ of $V$ into independent sets (in which some of the $A_{j}$ may be empty); the $k$ sets $A_{j}$ are called the color classes of the $k$-coloring. The chromatic number $\chi(G)$ is the minimum value $k$ for which a $k$-coloring exists. A $k$-coloring $A_{1}, A_{2}, \ldots, A_{k}$ is $\ell$-bounded if $\left|A_{i}\right| \leq l, 1 \leq i \leq k$. A $k$-coloring $A_{1}, A_{2}, \ldots, A_{k}$ is equitable if for any $i, j \in\{1,2, \ldots, k\},\left|A_{i}-A_{j}\right| \leq 1$.

Theorem 1.4 ([21]). Every $n$-vertex d-degenerate graph $G$ is equitably $k$-colorable for any $k \geq \max \left\{62 d, 31 d \frac{n}{n-\Delta(G)+1}\right\}$.

Every graph of treewidth $\leq d$ is $d$-degenerate and Theorem 1.4 implies the following corollary.

Corollary 1.5. Every $n$-vertex graph $G$ of treewidth $w$ is equitably $k$-colorable for any $k \geq \max \left\{62 w, 31 w \frac{n}{n-\Delta(G)+1}\right\}$.

\section{Covering by equitable independent sets.}

Let $S \subseteq V$ be a set of vertices of a graph $G=(V, E)$. We say that $S$ can be covered by independent sets of sizes $[n / k]$ if there is a set of subsets $A_{i} \subseteq V, i \in\{1,2, \ldots, p\}, p \leq|S|$, such that

(i) For every $i \in\{1,2, \ldots, p\}, A_{i}$ is an independent set;

(ii) For every $i, j \in\{1,2, \ldots, p\}, i \neq j, A_{i} \cap A_{j}=\emptyset$;

(iii) For every $i \in\{1,2, \ldots, p\}$, either $\left|A_{i}\right|=\lceil n / k\rceil$, or $\left|A_{i}\right|=\lfloor n / k\rfloor$;

(iv) $S \subseteq \cup_{1 \leq i \leq p} A_{i}$.

Covering by independent sets is a natural generalization of equitable coloring: A graph $G$ has equitable $k$-coloring if and only if $V$ can be covered by independent sets of sizes $[n / k]$. We use the following observations in our proof.

Lemma 2.1. Let $S \subseteq V$ be a vertex subset of a graph $G$.

(a) If $S$ can not be covered by independent sets of sizes $[n / k]$, the graph $G$ is not equitable $k$-colorable;

(b) If $S$ can be covered by $p$ independent sets $A_{1}, \ldots, A_{p}$ of sizes $[n / k]$ and the graph $G^{\prime}=G\left[V-\cup_{1 \leq i \leq p} A_{i}\right]$ is equitable $(k-p)$-colorable, the graph $G$ is equitable $k$ colorable.

Let $G$ be a graph of treewidth $w$. The next theorem implies that when the cardinality of $S \subseteq V$ or the number $k$ is at most $f(w)$, where $f$ is a function of $w$, the question if $S$ can be covered by independent sets of sizes $[n / k]$ can be answered in polynomial time. Because there are graphs that need $\Omega(n)$ colors in an equitable coloring, Theorem 2.2 does not imply directly that for graphs of bounded treewidth the EQUITABLE COLORING problem can be solved in polynomial time. 
Theorem 2.2. Let $G=(V, E)$ be an n-vertex graph of treewidth $\leq w$, let $S$ be a subset of $V$, and let $k$ be an integer. One can either find a covering of $S$ by independent sets of sizes $[n / k]$, or conclude that there is no such a covering in polynomial time when $k$ is bounded by a constant, or when $|S|$ is bounded by a constant.

Proof. This can be shown using standard dynamic programming techniques for graphs of bounded treewidth. Note that we can check for a covering of at most $\min \{k,|S|\}$ independent sets of sizes $[n / k]$. An algorithm comparable to those e.g. shown in $[6,27]$, that also has different table entries / homomorphism classes when sets have different sizes solves the problem in polynomial time on graphs of bounded treewidth.

\section{Bounded treewidth}

The main result of this paper is the following theorem.

Theorem 3.1. Equitable k-colorability problem can be solved in polynomial time on graphs of bounded treewidth.

Proof. Let $G=(V, E)$ be a graph of treewidth $w$ and let $k$ be an integer. To determine if $G$ has an equitable $k$-coloring, we consider the following cases.

Case 1. $\Delta(G) \leq n / 2+1$ and $k \geq 62 w$. Since

$$
\max _{0 \leq \Delta(G) \leq n / 2+1} \frac{n}{n-\Delta(G)+1}=2,
$$

we have that

$$
k \geq 62 w=\max \{62 w, 2 \cdot 31 w\} \geq \max \left\{62 w, 31 w \frac{n}{n-\Delta(G)+1}\right\}
$$

and by Corollary $1.5, G$ is equitably $k$-colorable.

Case 2. $\Delta(G) \leq n / 2+1$ and $k \leq 62 w$. In this case, it follows from Theorem 2.2 that the question whether $G$ has an equitably $k$-coloring can be solved in polynomial time.

Case 3. $\Delta(G)>n / 2+1$. Let $S \subset V$ be the set of vertices in $G$ of degree $\geq n / 2+2$. By Lemma 1.3, $G$ has at most $w n$ edges, so $|S| \leq 4 w$. Thus by Theorem 2.2 , it can be checked in polynomial time whether $S$ can be covered by independent sets of sizes $[n / k]$. If $S$ cannot be covered, by part (a) of Lemma 2.1, $G$ has no equitable $k$-coloring.

Let $A_{i} \subset V, i \in\{1,2, \ldots, p\}, p \leq|S|$, be an equitable covering of $S$ by independent sets of sizes $[n / k]$. We define a new graph $G^{\prime}=G\left[V-\cup_{1 \leq i \leq p} A_{i}\right]$. The maximum vertex degree in $G^{\prime}$ is at most $n / 2+1$ and the treewidth of $G^{\prime}$ is $\leq w$. Graph $G^{\prime}$ has

$$
n^{\prime}=\left|V-\cup_{1 \leq i \leq p} A_{i}\right| \geq n-p\left\lfloor\frac{n}{k}\right\rfloor \geq n-4 w\left(\frac{n}{k}-1\right)>\left(1-\frac{4 w}{k}\right) n
$$

vertices. 
Let $k^{\prime}=k-p$. We need again case distinction.

Case A. $k^{\prime} \geq \max \left\{62 w, 31 w \frac{n^{\prime}}{n^{\prime}-n / 2}\right\}$. Then

$$
k^{\prime} \geq \max \left\{62 w, 31 w \frac{n^{\prime}}{n^{\prime}-n / 2}\right\} \geq \max \left\{62 w, 31 w \frac{n^{\prime}}{n^{\prime}-\Delta\left(G^{\prime}\right)+1}\right\}
$$

and by Corollary 1.5, $G^{\prime}$ is equitably $k^{\prime}$-colorable. By part (b) of Lemma 2.1, $G$ has equitable $k$-coloring.

Case B. $k^{\prime}<\max \left\{62 w, 31 w \frac{n^{\prime}}{n^{\prime}-n / 2}\right\}$ and $k^{\prime}<62 w$. Since $p \leq 4 w$, we have that $k=$ $k^{\prime}+p<66 w$. Then by Theorem 2.2 , the question whether $G$ has an equitably $k$-coloring, can be solved in polynomial time.

Case C. $k^{\prime}<\max \left\{62 w, 31 w \frac{n^{\prime}}{n^{\prime}-n / 2}\right\}$ and $k^{\prime} \geq 62 w$. Then

$$
k^{\prime}<31 w \frac{n^{\prime}}{n^{\prime}-\frac{n}{2}}<31 w \frac{n}{\left(1-\frac{4 w}{k}\right) n-\frac{n}{2}}=\frac{31 w}{\frac{1}{2}-\frac{4 w}{k}} .
$$

Using $k=k^{\prime}+p \geq 62 w$, we have that

$$
\frac{4 w}{k} \leq \frac{4 w}{62 w}=\frac{2}{31}
$$

and

$$
\frac{1}{2}-\frac{4 w}{k} \geq \frac{27}{62}
$$

By (1) and (2),

$$
k^{\prime}<31 w \frac{62}{27}<72 w
$$

and we conclude that $k=k^{\prime}+p \leq 76 w$. Again, by Theorem 2.2 the question if $G$ has an equitably $k$-coloring, can be solved in polynomial time.

By Proposition 1.1, Theorem 3.1 implies directly that there is a polynomial time algorithm for the $\ell$-BOUNDED COLORING problem restricted to graphs of bounded treewidth.

\section{Equitable coloring with pre-coloring}

For a graph $G$, a pre-coloring $p$ of a subset $V^{\prime} \subset V$ in $k$ colors is a mapping $\pi: V^{\prime} \rightarrow$ $\{1,2, \ldots, p\}$. We say that a coloring $A_{1}, A_{2}, \ldots, A_{m}$ of $G$ extends the pre-coloring $\pi$ if $u \in$ $A_{\pi(u)}$ for every $u \in V^{\prime}$. We consider the following problem: EQUiTABLE COLORING WiTH PRE-COLORING: For a given graph $G$, integer $k$ and a given pre-coloring $\pi$ of $G$, determine the smallest integer $k$ for which there exists an equitable $k$-coloring of $G$ extending $\pi$.

Theorem 4.1. Equitable COLORING With PRE-COLORING is NP hard on forests. 
Proof. We use a reduction from the problem

\section{3-PARTITION}

Instance: A set $A$ of non-negative integers $a_{1}, \ldots, a_{3 m}$, and a bound $B$, such that for all $i$ with $1 \leq i \leq 3 m,(B+1) / 4<a_{i}<B / 2$ and $\sum_{1 \leq i \leq 3 m} a_{i}=m B$.

Question: Can $A$ be partitioned into $m$ disjoint sets $A_{1}, A_{2}, \ldots, A_{m}$ such that $\sum_{a_{i} \in A_{j}} a_{i}=B$ for every $j$ with $1 \leq j \leq m$ ?

3-PARTITION is NP-complete in the strong sense (Problem SP15 in Garey \& Johnson [12]).

Let the set $A=\left\{a_{1}, \ldots, a_{3 m}\right\}$ and the bound $B$ be an instance of 3-PARTition. We construct a forest $G$ and pre-coloring of $G$ such that $G$ is equitable $(m+1)$-colorable if and only if $A$ can be 3 -partitioned.

For every $i \in\{1,2, \ldots, m\}$ we define the set $N_{i}=\{1,2, \ldots, m\}-\{i\}$ and pre-colored star $S_{i}$ as a star with one non-pre-colored central vertex $v$ adjacent to $m-1$ leaves which are pre-colored in all colors from $N_{i}$. Thus vertex $v$ can be colored only in color $i$ or $m+1$. For every $i \in\{1,2, \ldots, m\}$ and $j \in\{1,2, \ldots, 3 m\}$, we define the pre-colored tree $G_{i, j}$ as a tree obtained by taking the disjoint union of $a_{j}+1$ pre-colored stars $S_{i}$ and by making the central vertex $v$ of one of them to be adjacent to the central vertices of the others $a_{j}$ stars. We call the vertex $v$ the central vertex of $G_{i, j}$. Thus $G_{i, j}$ has $m\left(a_{j}+1\right)$ vertices; for every color $\ell \in N_{i}$ there are $\left(a_{j}+1\right)$ vertices of $G_{i, j}$ pre-colored by $\ell$. Every $(m+1)$-coloring of $G_{i, j}$ either colors $v$ in $m+1$ and remaining $a_{j}$ non-pre-colored vertices in $i$, or it colors $v$ in $i$ and remaining $a_{j}$ non-pre-colored vertices in $m+1$. (See Fig. 1.)

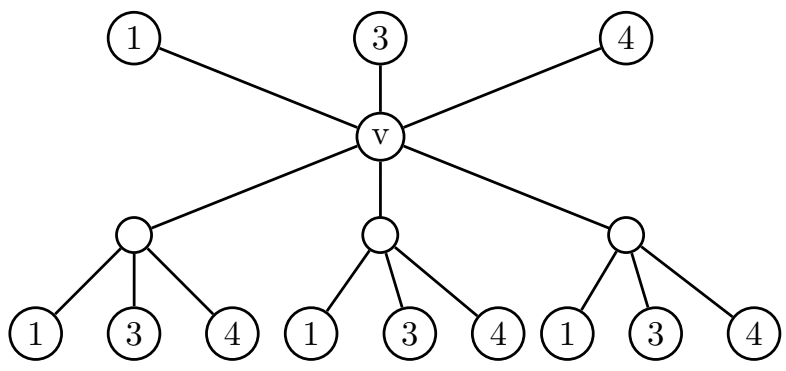

Figure 1: Tree $G_{i, j}$. Here $a_{j}=3, m=4, i=2$ and $N_{2}=\{1,3,4\}$. In any 5-coloring of $G_{i, j}$, either $v$ should be colored by 5 and all its non pre-colored neighbors by 2 , or $v$ should be colored by 2 and the neighbors by 5 .

For every $j \in\{1,2, \ldots, 3 m\}$, we define a pre-colored tree $G_{j}$ as follows: We take the disjoint union of pre-colored trees $G_{i, j}, i \in\{1,2, \ldots, m\}$, add one vertex $c_{j}$ adjacent to all central vertices of trees $G_{i, j}$ and add one leaf adjacent to $c_{j}$ pre-colored in $m+1$. Thus $G_{j}$ has $m^{2}\left(a_{j}+1\right)+2$ vertices; for every color $\ell \in\{1,2, \ldots, m\}-\{i\}$ there are $(m-1)\left(a_{j}+1\right)$ vertices of $G_{j}$ pre-colored by $\ell$ and there is 1 vertex pre-colored by $m+1$. Also in any coloring of $G_{j}$ vertex $c_{j}$ can not be colored in $m+1$. Thus for every $(m+1)$-coloring of $G_{j}$ the spectra of colors used on neighbors of $c_{j}$ does not contain all colors from $\{1,2, \ldots, m\}$. 
Finally, the forest $G$ is the disjoint union of pre-colored trees $G_{1}, G_{2}, \ldots, G_{3 m}$ and independent set of cardinality $3 m(m-2)+B$ pre-colored in color $m+1$. Thus $G$ has

$$
\sum_{1 \leq j \leq 3 m} m^{2}\left(a_{j}+1\right)+3(m-2)+B=m^{2}(m B+3 m)+3(m-2)+B
$$

vertices. For every color $\ell \in\{1,2, \ldots, m\}-\{i\}$ there are

$$
\sum_{1 \leq j \leq 3 m}(m-1)\left(a_{j}+1\right)=(m-1)(m B+3 m)
$$

vertices of $G$ pre-colored by $\ell$ and $3 m(m-1)+B$ vertex are pre-colored by $m+1$.

Suppose that $A$ can be partitioned into $m$ disjoint sets $A_{1}, A_{2}, \ldots, A_{m}$ such that $\sum_{a_{i} \in A_{j}} a_{i}=B$. We define an extension of pre-coloring of $G$ as follows. For every fixed $j \in\{1,2, \ldots, 3 m\}$, we choose $i$ such that $a_{j} \in A_{i}$. We color central vertex of $G_{i, j}$ in color $m+1$ and the remaining noncolored $a_{j}$ vertices of $G_{i, j}$ in color $i$. In each graph $G_{\ell, j}, \ell \neq i$, $a_{j}$ vertices are colored by $m+1$ and one vertex by $\ell$. Also we color vertex $c_{j}$ with $i$. Thus in every graph $G_{j}$ on the set of non-pre-colored vertices color $i$ is used $a_{j}+1$ times. Any color $\ell \in\{1,2, \ldots, m\}-\{i\}$ is used one time and color $m+1$ is used

$$
a_{j}(m-1)+1
$$

times on non-pre-colored vertices. Thus in graph $G$ the number of vertices colored by color $\ell \in\{1,2, \ldots, m\}$ is

$$
(m-1)(m B+3 m)+\sum_{a_{j} \in A_{\ell}}\left(a_{j}+1\right)+\sum_{\left\{1 \leq j \leq 3 m \mid a_{j} \notin A_{\ell}\right\}} 1=(m-1)(m B+3 m)+B+3 m .
$$

The number of vertices colored in $m+1$ is

$$
3 m(m-2)+B+\sum_{1 \leq j \leq 3 m}\left(a_{j}(m-1)+1\right)=(m-1)(m B+3 m)+B+3 m
$$

and we conclude that the obtained coloring is equitable $(m+1)$-coloring.

Suppose now that $G$ is equitable $(m+1)$-colorable. The main observation here is that for every $j \in\{1,2, \ldots, 3 m\}$ at most $a_{j}(m-1)+1$ vertices of a graph $G_{j}$ are colored by $m+1$. (Otherwise coloring of central vertices of graphs $G_{i, j}, i \in\{1,2, \ldots, m\}$, uses the whole spectra $\{1,2, \ldots, m\}$ thus leaving no space for color of $c_{j}$.) If for some $j \in\{1,2, \ldots, 3 m\}$ less than $a_{j}(m-1)+1$ vertices of a graph $G_{j}$ are colored with color $m+1$ then (the coloring is equitable) for some $j^{\prime} \in\{1,2, \ldots, 3 m\}$ at least $a_{j} m$ vertices of graph $G_{j^{\prime}}$ are colored by $m+1$, which is a contradiction.

Thus we can conclude that for every $j \in\{1,2, \ldots, 3 m\}$ there is exactly one subgraph $G_{i, j}$ such that $a_{j}$ non-pre-colored vertices of $G_{i, j}$ are colored with $i$. For all other $i^{\prime} \in$ $\{1,2, \ldots, m\}, i \neq i^{\prime}, a_{j}$ non-pre-colored vertices of $G_{i^{\prime}, j}$ are colored with $m+1$. We define

$$
A_{i}=\left\{a_{j}: a_{j} \text { non-pre-colored vertices of } G_{i, j} \text { are colored with } i\right\} .
$$


In $G$ the number of vertices colored by color $i \in\{1,2, \ldots, m\}$ is

$$
(m-1)(m B+3 m)+B+3 m=(m-1)(m B+3 m)+\sum_{a_{j} \in A_{i}}\left(a_{j}+1\right)+\sum_{\left\{1 \leq j \leq 3 m \mid a_{j} \notin A_{i}\right\}} 1 .
$$

Thus for every $i \in\{1,2, \ldots, m\}$

$$
\sum_{a_{j} \in A_{i}}\left(a_{j}+1\right)=B
$$

and $A_{1}, A_{2}, \ldots, A_{m}$ is a 3 -partition of $A$.

So we have a polynomial reduction from 3-PARTITION to EQUITABLE COLORING WITH PRE-COLORING. As EQUITABLE COLORING WITH PRE-COLORING trivially belongs to NP, we can conclude it is NP-complete.

\section{References}

[1] N. Alon, A note on the decomposition of graphs into isomorphic matchings, Acta Math. Hungar., 42 (1983), pp. 221-223.

[2] B. S. Baker And E. G. Coffman, JR., Mutual exclusion scheduling, Theoret. Comput. Sci., 162 (1996), pp. 225-243.

[3] J. Blazewicz, K. H. Ecker, E. Pesch, G. Schmidt, and J. Weglarz, Scheduling Computer and Manufacturing Processes, Springer, Berlin, 2001. 2nd ed.

[4] H. L. Bodlaender And K. JAnsen, Restrictions of graph partition problems. I, Theoret. Comput. Sci., 148 (1995), pp. 93-109.

[5] B. BollobÁs And R. K. GuY, Equitable and proportional coloring of trees, J. Combin. Theory Ser. B, 34 (1983), pp. 177-186.

[6] R. B. BoRIE, Generation of polynomial-time algorithms for some optimization problems on tree-decomposable graphs, Algorithmica, 14 (1995), pp. 123-137.

[7] G. J. Chang And D. Kuo, The L(2,1)-labeling problem on graphs, SIAM J. Discrete Math., 9 (1996), pp. 309-316.

[8] B.-L. Chen, M.-T. Ko, And K.-W. LiH, Equitable and m-bounded coloring of split graphs, in Combinatorics and computer science (Brest, 1995), vol. 1120 of Lecture Notes in Comput. Sci., Springer, Berlin, 1996, pp. 1-5.

[9] B.-L. Chen And K.-W. LiH, Equitable coloring of trees, J. Combin. Theory Ser. B, 61 (1994), pp. 83-87. 
[10] E. Cohen And M. TARsi, NP-completeness of graph decomposition problems, J. Complexity, 7 (1991), pp. 200-212.

[11] M. R. Garey, R. L. Graham, D. S. Johnson, and D. E. Knuth, Complexity results for bandwidth minimization, SIAM J. Appl. Math., 34 (1978), pp. 477-495.

[12] M. R. Garey And D. S. Johnson, Computers and Intractability, A guide to the theory of NP-completeness, W. H. Freeman and Co., San Francisco, Calif., 1979.

[13] A. Hajnal and E. Szemerédi, Proof of a conjecture of P. Erdős, in Combinatorial theory and its applications, II (Proc. Colloq., Balatonfüred, 1969), North-Holland, Amsterdam, 1970, pp. 601-623.

[14] P. Hansen, A. Hertz, And J. Kuplinsky, Bounded vertex colorings of graphs, Discrete Math., 111 (1993), pp. 305-312. Graph theory and combinatorics (MarseilleLuminy, 1990).

[15] S. IRANi AND V. LeUnG, Scheduling with conflicts, and applications to traffic signal control, in Proceedings of the Seventh Annual ACM-SIAM Symposium on Discrete Algorithms (Atlanta, GA, 1996), New York, 1996, ACM, pp. 85-94.

[16] K. JANsEn, The mutual exclusion scheduling problem for permutation and comparability graphs, Information and Computation, 180 (2003), pp. 71-81.

[17] M. Jarvis And B. Zhou, Bounded vertex coloring of trees, Discrete Math., 232 (2001), pp. 145-151.

[18] D. Kaller, A. Gupta, And T. Shermer, The $\chi_{t}$-coloring problem, in Symp. on Theoretical Aspects of Comp. Sc., STACS'95 (Munich, 1995), vol. 900 of Lecture Notes in Comput. Sci., Springer, Berlin, 1995, pp. 409-420.

[19] F. Kitagawa And H. Ikeda, An existential problem of a weight-controlled subset and its application to school timetable construction, Proceedings of the First Japan Conference on Graph Theory and Applications (Hakone, 1986), Discrete Math., 72 (1988), pp. 195-211.

[20] D. Kobler AND U. Rotics, Edge dominating set and colorings on graphs with fixed clique-width, Discrete Appl. Math., 126 (2003), pp. 197-221.

[21] A. V. Kostochka, K. Nakprasit, and S. V. Pemmaraju, Coloring d-degenarate graphs equitable. Manuscript, 2003.

[22] J. Krarup AND D. DE WerRA, Chromatic optimisation: limitations, objectives, uses, references, European J. Oper. Res., 11 (1982), pp. 1-19.

[23] K.-W. LiH, The equitable coloring of graphs, in Handbook of Combinatorial Optimization, Vol. 3, Kluwer Acad. Publ., Boston, MA, 1998, pp. 543-566. 
[24] Z. Lonc, On complexity of some chain and antichain partition problems, in Graphtheoretic concepts in computer science WG'91 (Fischbachau, 1991), vol. 570 of Lecture Notes in Comput. Sci., Springer, Berlin, 1992, pp. 97-104.

[25] B. Monien, The bandwidth minimization problem for caterpillars with hair length 3 is NP-complete, SIAM J. Algebraic Discrete Methods, 7 (1986), pp. 505-512.

[26] B. F. Smith, P. E. Bjørstad, And W. D. Gropp, Domain Decomposition. Parallel multilevel methods for elliptic partial differential equations, Cambridge University Press, Cambridge, 1996.

[27] J. A. Telle And A. Proskurowski, Algorithms for vertex partitioning problems on partial k-trees, SIAM J. Discrete Math., 10 (1997), pp. 529 - 550. 